\title{
Fenômeno da hipercoagulabilidade em pacientes com COVID-19
}

Igor Gomes de Araújo ${ }^{1}$

Arlandia Cristina Lima Nobre de Morais²

\section{RESUMO}

O SARS-CoV-2 é o vírus responsável pela infecção respiratória do trato inferior, também conhecido como síndrome respiratória aguda grave. Considerando as manifestações clínicas graves, estima-se que 20-30\% apresentarão complicações a nível cardiovascular e 30-50\% a nível renal. Inicialmente o SARS-CoV-2 infecta as nossas células através de receptor da enzima conversora de angiotensina 2 (ECA2 ou ACE-2), dos alvéolos pulmonares. Ocorrendo sensibilização de células de defesa com consequente liberação de citocinas no sangue, intitulada para a patologia como "tempestade de citocinas", entre elas, as interleucinas 1 e 6 (pró-inflamatórias), fator de necrose tumoral e interferon- $\rrbracket$. Observa-se nesta patologia a ocorrência de eventos de hipercoagulabilidade e isquemia decorrente da hipóxia. Para então reverter essa condição clínica, vem surgindo a hipótese do uso de anticoagulantes na fisiopatologia de pacientes graves com COVID-19. Após resposta imunológica ocorre o evento inflamatório com consequentes danos endoteliais. Os mediadores da inflamação citados anteriormente ativam fatores procoagulantes. Entres eles, fatores extrínsecos e intrínsecos. Partindo de tal premissa, todos os pacientes hospitalizados com COVID-19 devem receber anticoagulantes caso não apresente riscos de sangramento. No entanto, faz-se necessário estudos randomizados com a heparina não fracionada e de baixo peso molecular para comprovação da atividade inibidora do SARS-CoV-2, além de apresentar-se como perspectiva em ocasionarem modificações conformacionais no vírus, tornando-as uma possibilidade farmacológica. A partir dos estudos analisados pôde-se concluir que o emprego de anticoagulantes na fisiopatologia da COVID-19 são bastante eficazes, com relação ao mecanismo de hipercoagulabilidade que a doença promove.

Palavras-chave: hipercoagulabilidade; SARS-CoV-2; COVID-19; heparina.

\section{ABSTRACT}

SARS-CoV-2 is the virus responsible for respiratory infection of the lower tract, also known as severe acute respiratory syndrome. Considering the severe clinical manifestations, it is estimated that 20-30\% will have complications at the cardiovascular level and 30-50\% at the renal level. Initially, SARS-CoV-2 infects our cells through an angiotensin-converting enzyme receptor 2 (ECA2 or ACE-2) in the pulmonary alveoli. There is sensitization of defense cells with consequent release of cytokines in the blood, entitled for the pathology as "cytokine storm", including interleukins 1 and 6 (proinflammatory), tumor necrosis factor and $\bigotimes$-interferon. It is observed in this pathology the occurrence of events of hypercoagulability and ischemia due to hypoxia. Then, to reverse this clinical condition, the hypothesis of the use of anticoagulants in the pathophysiology of critically ill patients with COVID-19 has been emerging. After an immunological response, an inflammatory event occurs with consequent endothelial damage. The inflammation mediators mentioned above activate procoagulating factors. Among them, extrinsic and intrinsic factors. Based on this premise, all patients hospitalized with COVID-19 should receive anticoagulants if they are not at risk of bleeding. However, randomized studies with unfractionated and low molecular weight heparin are necessary to prove the inhibitory activity of SARS-CoV-2, in addition to presenting a perspective on causing conformational changes in the virus, making them a possibility pharmacological. From the studies analyzed, it was concluded that the use of anticoagulants in the pathophysiology of COVID-19 is quite effective, in relation to the hypercoagulability mechanism that the disease promotes.

Keywords: hypercoagulability; SARS-CoV-2; COVID-19; heparin.

\footnotetext{
${ }^{1}$ Universidade de Fortaleza, Avenida Washington Soares, 1321, Edson Queiroz, CEP 60811-905, Fortaleza, Ceará, Brasil. E-mail: gomes.igor1996@gmail. com

${ }^{2}$ Universidade de Fortaleza, Avenida Washington Soares, 1321, Edson Queiroz, 60811-905, Fortaleza, Ceará, Brasil.

Como citar este artigo / How to cite this article

de Araujo IG, de Morais ACLN. Fenômeno da hipercoagulabilidade em pacientes com COVID-19. InterAm J Med Health 2020;3:e202003037.
} 


\section{INTRODUÇÃO}

O SARS-CoV-2 é o vírus responsável pela infecção respiratória do trato inferior, também conhecido como síndrome respiratória aguda grave Trata-se de uma pneumonia decorrente da infeccão virat, com uma pneumonia decorente da infeccá, viral com comprometimento deste importante órgão, que possui função de absorver oxigênio (O2) nos alvéolos e eliminar dióxido de carbono (CO2). Cerca de $20 \%$ das pessoas infectadas necessitam de internamento e entre 5-10\% de unidade de terapia intensiva (UTI), para suportes hemodinâmicos e ventilatórios [1]

O agravamento das manifestações clínicas ocorrem entre o $7^{\circ}$ ao $12^{\circ}$ dia após o aparecimento dos primeiros sintomas. De maior gravidade para pacientes que apresentam hipoxemia e infiltrado pulmonar bilateral. Considerando este cenário grave, estima-se que 20-30\% apresentarão complicações a nível cardiovascular e 30$50 \%$ a nível renal [1-2]

Inicialmente o SARS-CoV-2 infecta as nossas células através de receptor da enzima conversora de angiotensina 2 (ECA2 ou ACE-2), dos alvéolos pulmonares. Ocorrendo sensibilização de células de defesa com consequente liberação de citocinas no sangue, intitulada para a patologia como "tempestade de citocinas", entre elas, as interleucinas 1 e 6 (pró-inflamatórias), fator de necrose tumoral e interferon- $₫[1-3]$

Observa-se nesta patologia a ocorrência de eventos [4-5].

Para então reverter essa condição clínica, vem surgindo a hipótese do uso de anticoagulantes na fisiopatologia de pacientes graves com COVID-19. Sabese que há uma ativação da coagulação ocasionando formação de coágulos e trombos a nível pulmonar e cerebral.

A hemostasia é um sistema complexo cujo mecanismo é promover o equilíbrio, impedindo que ocorra hemorragia, sincronicamente evitando a formação de trombos, consequências de pouca formação e muita formação de fibrina, respectivamente. Neste sistema hemostático incluem as plaquetas, os vasos, as proteínas da coagulação sanguínea, os anticoagulantes fisiológicos e o sistema de fibrinólise [6]

Após resposta imunológica ocorre o evento inflamatório com consequentes danos endoteliais. Os mediadores da inflamação citados anteriormente ativam fatores procoagulantes. Entre eles, fatores extrinsecos, fator XVII ativa fator Xe intrínsecos, ativação de fator XI quando o sangue entra em contato com superfície que possui cargas elétricas negativas, possuindo presença de pré-calicreína (seriprotease) e cininogênio (cofator não enzimático). O fator Xlla ativa o fator Xl, que ativa fator X. Por sua vez, o fator IXa na companhia do fator VIII, fibrinoghio converida en fribmbina, que onghara comvertida em fibrina, proteina fisiológica cong plasminogênio. Estas reaçôes ocorrem em superfícies com fosfolipídios de membrana [1-6].

O estudo de Chen et al (2020), relacionou a letalidade da doença em pacientes com excessiva concentração do dímero- $D$, produto de degradação da fibrina [7]. O dímero-D é dosado em pacientes com suspeita de tromboembolismo pulmonar (TEP) e trombose venosa profunda (TVP), pelo método ELISA quantitativo, tornando-se um exame vantajoso e com alta sensibilidade (acima de 99\%). Os níveis elevados do dímero-D (acima de $500 \mathrm{ng} / \mathrm{mL}$ ) também são encontrados nas seguintes situações: infarto agudo do miocárdio, sepses, neoplasias, pós-operatórios (até 1 semana), coagulação intravascular disseminada, anemia falciforme, insuficiência cardíaca e pneumonias [8].

Observa-se ao analisar a provável fisiopatologia da COVID-19 uma diminuição da fibrinólise, resultando na diminuição do tempo de protrombina (PT), fator importante na coagulação sanguínea, favorecendo assim a capacidade em formação de coágulos e trombos [9].

O tempo de protrombina (PT), foi especificado por Quick, em 1935, com propósito em quantificar a protrombina e avaliar os fatores $\|, \mathrm{V}, \mathrm{V}\| \mathrm{X}$, vias extrínsecas da coagulação [10].

Partindo de tal premissa, todos os pacientes hospitalizados com COVID-19 devem receber anticoagulantes caso não apresente riscos de sangramento. No estudo de Mycroft-West et al (2020), concluíram que a heparina foi capaz de inibir entre 44 e $80 \%$ o SARSCov-2 em células de macacos. A heparina foi capaz de ligar-se ao receptor de proterna Spike (S1) e a enoxa à proteína $\mathrm{S} 1 \mathrm{RBD}$, ambas capazes de induzir alteração conformacional [11]

Neste contexto, Tang et al (2020), realizaram um estudo com 449 pacientes graves com COVID-19, dentre os quais 99 receberam terapia com heparina (principalmente de baixo peso molecular) por 7 ou mais dias. Não foi encontrado diferença na mortalidade em 28 dias em pacientes que receberm heprina ñ̃o usú́rios $(30,3 \%$ vs $29,7 \%, \mathrm{P}=0,910)$. Todavia, a mortalidade em 28 dias foi menor em usuários de heparina com pontuação SIC relação ao mecanismo de hipercoagulabilidade que a $\geq 4(40,0 \%$ vs $64,2 \%, P=0,029)$ ou $D$-dímero> 6 vezes o doença promove.

limite superior do normal $(32,8 \%$ vs $52,4 \%, P=0,017)$.

A pontuação SIC é uma sigla em inglês que se traduz por

"coagulopatia induzida por sepse", sendo considerado

os parâmetros tempo de protrombina, contagem de plaquetas e avaliação sequencial de falência de órgãos (SOFA). Concluindo que parece estar associada a um melhor prognóstico [12]

A Associação de Medicina Intensiva Brasileira, a Sociedade Brasileira de Infectologia e a Sociedade Brasileira de Pneumologia e Tisiologia, publicaram diretrizes para o tratamento farmacológico da COVID-19 do qual, há recomendação forte no uso de heparina para do qual, há recomendação forte no uso de heparina para fins de profilaxia para tromboembolismo venoso de rotina, com recomendação fraca do uso em dose terapêutica (ex: enoxaparina $1 \mathrm{mg} / \mathrm{kg}$ subcutânea [SC] a cada 12 horas), ambas recomendações ainda possuem nível de evidência estudo de Tang et al (2020) [12]. Seguido da analogia para outros anticoagulantes. Todavia, a dose profilática poderá ser empregada ser empregada em pastúrbios nos fatores de coagulação (ex: enoxaparina 40 a $60 \mathrm{mg}$ SC $1 \times$ ao dia ou a heparina não fracionada 5.000UI SC 2 a $3 x$ ao dia) [13], mediante eventos observados em estudos clínicos observacionais e post mortem [14-15]. Salientando que a heparina de baixo peso molecular (HBPM) deve ser administrada com atenção em pacientes com disfunção renal [13].

No estudo de Han et al (2020), 94 pacientes com infecção confirmada por SARS-CoV-2 tiveram os níveis de antitrombina analisados. Observou-se que esses níveis foram inferiores aos do grupo controle. Também os valores do dímero $D$, produtos de degradação de fibrina/ fibrinogênio (FDP) e fibrinogênio (FIB) em todos os casos de SARS-CoV-2 foram substancialmente mais altos do que os de controles saudáveis. Dessa forma, concluíram que é significativamente acometida em comparação com as essoas saudáveis [16]

No entanto, faz-se necessário estudos randomizados com a heparina não fracionada e de baixo peso molecular para comprovação da atividade inibidora do SARS-CoV-2, além de apresentar-se como perspectiva em ocasionarem modificações conformacionais no vírus, tornando-as uma possibilidadefarmacológica. A partirdos estudos analisados fisiopatologia da COVID-19 são bastante eficazes, com

\section{REFERENCIAS}

1. Nascimento JPH; Gomes BFO; Júnior PRC; Petriz JLF; Rizk Sl; Costa IBSS et al. COVID-19 e estado de hipercoagulabilidade: Uma nova perspectiva terapêutica. Arq Bras Cardiol. 2020;114(5):829-33. Disponível em: http:// publicacoes cardiol.br/portal/abc/portugues/2020/v11405/ pdf/11405016.pdf. Acesso em: 23 de maio de 2020.

2. Wang D; Hu B; Hu C; Zhu F; Liu X; Zhang J et al. Clinical Characteristics of 138 Hospitalized Patients With 2019 Novel Coronavirus-Infected Pneumonia in Wuhan, China. JAMA. 2020;323(11):1061-69. Disponivel em: https://jamanetwork.com/journals/jama/ fullarticle/2761044. Acesso em: 23 de maio de 2020.

3. Chen G. Wu D. Guo W. Li X. Sun Y. Li I et al I Clinical and immunologic features in severe and moderate Coronavirus Disease 2019. J Clin Invest. 2020:130(5):262029. Disponivel em: https://pubmed.ncbi.nlm.nih. gov/32217835/. Acesso em: 23 de maio de 2020. 4. Zhang T; Sun LX; Feng RE. Comparison of clinical and pathological features between severe acute respiratory syndrome and coronavirus disease 2019. Zhonghua Jie He He Hu Xi Za Zhi. 2020:43. Disponível em: https:/pubmed nchi. Im.nih.gov/322410721. Acesso em: 23 de maio de 2020. 5. Agência Fapesp. Autópsia em mortos por COVID-19 ajuda no tratamento de casos graves da doença. Disponível em: $\quad$ http://agencia.fapesp.br/autopsia-em-mortospor-covid-19-ajuda-no-tratamento-de-casos-gravesda-doenca/32882/. Acesso em: 23 de maio de 2020.

6ranco RF. Fisiologia da coagulação, anticoagulação e fibrinólise. Medicina, Ribeirão Preto. 2001;34:229-237. Disponível em: http///revista.fmrp.usp br/2001/vol34n3e4/ fisiologia_coagulacao.pdf. Acesso em: 23 de maio de 2020. 7. Chen N; Zhou M; Dong X; Qu J; Gong F; Han Y et al. Epidemiological and clinical characteristics of 99 cases of 2019 novel coronavirus pneumonia in Wuhan, China: a descriptive study. Lancet. 2020;395(10223):507ه13. Disponível em: https://pubmed.ncbi.nlm.nih. gov/32007143/. Acesso em: 23 de maio de 2020. 
8. Monachini M. Qual o valor do dímero d no diagnóstico do tromboembolismo pulmonar? Rev Assoc Med Bras. 2002;3(48). Disponível em: https://www.scielo.br/scielo. php ? pid=S0104-42302002000300012\&script $=$ sci_ arttext. Acesso em: 24 de maio de 2020.

9. Brandão SCS; Silva ETAGBB; Ramos JOX; Melo LMMP; Sarinho ESC. COVID-19, imunidade, endotélio e coagulação: compreenda a interação. 2020.

10. Reis PRM; Mesquita MM; Dias-Penna KGB; Castro FS; Balestra FM. Avaliação da determinação do tempo de protrombina em amostras de sangue colhidas por duas diferentes técnicas. J Bras Patol Med Lab. 2005;4(41):2515. Disponível em: https://www.scielo.br/pdf/jbpml/ v41n4/a06v41n4.pdf. Acesso em: 24 de maio de 2020.

11. Mycroft-West CJ; Su D; Pagani I; Rudd TR; Elli S; Guimond SE et al. Heparin inhibits cellular invasion by SARS-CoV-2: structural dependence of the interaction of the surface protein (spike) S1 receptor binding domain with heparin. bioRxiv. 2020. Disponivel em: https:// www. biorxiv.org/content/10.1101/2020.04.28.0667 61v2.full.pdf+html. Acesso em: 23 de maio de 2020.

12. Tang N; Bai H; Chen X; Gong J; Li D; Sun Z. Anticoagulant Treatment Is Associated With Decreased Mortality in Severe Coronavirus Disease 2019 Patients With Coagulopathy. J Thromb Haemost. 2020;18(5):109499. Disponível em: https://pubmed.ncbi.nlm.nih. gov/32220112/. Acesso em: 23 de maio de 2020.

13. Sociedade Brasileira de Pneumologia e Tisiologia (SBPT). DiretrizesparaotratamentofarmacológicodaCOVID-19.2020. Disponívelem:https://sbpt.org.br/portal/diretrizes-tratamentofarmacologico-covid/. Acesso em: 24 de maio de 2020.

14. Carsana L; Sonzogni A; Nasr A; Rossi R; Pellegrinelli A; Zerbi P. Pulmonary post-mortem findings in a large series of COVID-19 cases from Northern Italy. medRxiv. 2020. Disponível em: https://www.medrxiv.org/content/10.1101/2 020.04.19.20054262v1. Acesso em: 24 de maio de 2020.

15. Klok FA; Kruip MJHA; Van Der Meers NJM; Arbous MS; Gommers DAMP;; Kant KM. Incidence of thrombotic complications incritically ill ICU patients withCOVID-19. Thromb Res. 2020. Disponível em: https://www.sciencedirect.com/science/article/pii/ S0049384820301201. Acesso em: 24 de maio de 2020.
16. Han H; Yang L; Liu R; Liu F; Wu KL; Li J. Prominent changes in blood coagulation of patients with SARS-CoV-2 infection. Clin Chem Lab Med. 2020. Disponível em: https://pubmed. ncbi.nlm.nih.gov/32172226/. Acesso em: 23 de maio de 2020. 